\title{
(6) OPEN ACCESS \\ Prosthetic replacement of the ocular surface ecosystem: impact at 5 years
}

\author{
Joshua S Agranat, ${ }^{1,2}$ Nicole R Kitos, ${ }^{3}$ Deborah S Jacobs ${ }^{1}$
}

${ }^{1}$ Boston Foundation for Sight, Needham, Massachusetts, USA ${ }^{2}$ Boston University School of Medicine, Boston,

Massachusetts, USA

${ }^{3}$ Department of Neurology, Massachusetts General Hospital, Boston, Massachusetts, USA

\section{Correspondence to} Joshua S Agranat, Boston Foundation for Sight, 464 Hillside Avenue \# 205, Needham, MA 02494, USA; Jagranat@bu.edu

Preliminary analyses of data reported in this manuscript were presented at the American Academy of Ophthalmology Annual Meeting, Chicago, October 2014, ARVO 2014 Annual Meeting, Orlando, May 2014 and Cornea Society/EBAA, New Orleans, November 2013.

Received 13 July 2015 Revised 23 September 2015 Accepted 8 November 2015 Published Online First 7 December 2015
CrossMark

To cite: Agranat JS, Kitos NR, Jacobs DS. Br J Ophthalmol

2016:100:1171-1175

\section{ABSTRACT}

Background/aims To determine the impact of prosthetic replacement of the ocular surface ecosystem (PROSE) treatment at 5 years.

Methods Retrospective review of clinical, manufacturing and quality databases at the Boston Foundation for Sight.

Results 121 patients who completed treatment and had 5-year follow-up data were identified from a cohort of patients ( $n=199)$ seen in consultation for PROSE treatment from January 2008 to June 2008. Mean age was 52 years, $M: F=56: 65$. The primary indication for treatment was ocular surface disease (OSD) in 64 patients and distorted corneal surface in 57 patients. At 5 years, continued device wear was confirmed in 89/121 (73.6\%) patients. Discontinuation of wear was confirmed in 32/121 (26.4\%). There was an increased likelihood of continued device wear at 5 years in patients with distorted cornea (84\%) compared with those with OSD $(64 \%),\left(p=0.0121, \chi^{2}\right)$. National Eye Institute Visual Function Questionnaire (NEI VFQ-25) composite score increased for patients wearing PROSE devices at 6 months $(\Delta=+23$ points, mean $=82$, $p<0.001$, twotailed t test) with no significant decline among those still wearing a device at 5 years $(\Delta=-4$ points, mean $=78$, $p=0.22$, two-tailed $t$ test). At 5 years, those wearing (mean=78) had a higher NEI VFQ-25 than those not wearing (mean $=70, p=0.029$, two-tailed t test).

Conclusions PROSE treatment offers continued benefit, as defined by improved visual function and continued device wear at 5 years, in patients with complex corneal disease. Patients with distorted cornea have a higher rate of continued wear at 5 years than patients with OSD, although this is not true among all subgroups within OSD.

\section{INTRODUCTION}

Prosthetic replacement of the ocular surface ecosystem (PROSE) treatment is an integrated and iterative medical treatment developed at Boston Foundation for Sight, 501(c)3, (Needham, Massachusetts, USA), to improve visual function in complex corneal disease. PROSE treatment uses Food and Drug Administration (FDA)-approved (1994) custom-designed and manufactured prosthetic devices to replace or support impaired ocular surface functions in cases of 'distorted corneal surface or certain ocular surface disorders'. The prosthetic devices used in PROSE treatment have been referred to in the past as Boston Scleral Contact Lenses, ${ }^{1}$ Boston Scleral Lenses, ${ }^{2}$ Boston Scleral Lens Prosthetic Devices, ${ }^{3}$ Boston Ocular Surface Prostheses (BOS-P), ${ }^{4}$ and are now known as BostonSight PROSE devices. During the treatment process, which typically encompasses $4-10$ days of office visits, prosthetic devices are custom designed and fabricated for the individual patient eye using a proprietary software system. PROSE devices support the primary functions of the ocular surface and cornea and are made of a rigid gas permeable fluorosilicone acrylate polymer (Equalens II, Bausch +Lomb, Bridgewater, New Jersey, USA). Each device incorporates custom design features that allow it to rest on the bulbar conjunctiva and vault the cornea without making any apical or peripheral corneal contact. A PROSE device is further characterised by minimal movement, fluid ventilation and a diameter of $17.5-23.0 \mathrm{~mm}$.

The clinical impact of PROSE treatment has been documented in a series of patients with a broad range of complex corneal disease ${ }^{245}$ as well as in reports on impact in specific ocular surface diseases (OSD) such as ocular chronic graft-versushost disease (GVHD), ${ }^{3} 6$ and Stevens-Johnson Syndrome, ${ }^{78}$ and in corneal ectasia which is a distorted corneal surface. ${ }^{9}$ Experience in the paediatric age group has also been reported. ${ }^{10}$ PROSE treatment has been shown to be cost effective in terms of health benefit to the patient. ${ }^{11}$ However, until this point, there have been no studies to examine the long-term effectiveness of PROSE treatment. The purpose of this retrospective study was to evaluate the success of PROSE treatment after 5 years in a cohort of 199 consecutive patients seen in consultation when measured by continued wear and impact on visual functioning at 5 years.

\section{METHODS}

This retrospective review underwent formal review and waiver by the New England Institutional Review Board. This review was conducted in compliance with Health Insurance Portability and Accountability Act Regulations and adhered to the tenets of the Declaration of Helsinki.

Databases of clinical, manufacturing and quality records at the Boston Foundation for Sight were queried concerning all patients seen in consultation for PROSE treatment from 1 January 2008 through 30 June $2008 \quad(n=199)$. Retrospective review of these records was undertaken. Records of patients that were classified as non-candidates at consultation or did not complete the customisation and training process $(n=47)$ and/or were deceased $(n=16)$ or lost to follow-up $(n=15)$ at 5 years were excluded from the final analysis, leaving 121 records for analysis. Disease severity was not a basis for exclusion. Patient age, sex, corneal disease, wearing status and visual functioning scores obtained using the NEI VFQ- $25^{12}$ at consultation, 6 months and 5 years were extracted. NEI VFQ-25 had been administered in person at initial 
consultation and by telephone at 6 months and 5 years. Device wear status at 6 months and 5 years was determined by data from prior telephone interview and/or review of medical and manufacturing records. The subjects in this cohort were grouped into those with OSD and those with distorted corneal surface as the primary indication for PROSE treatment.

Descriptive statistics were performed for several characteristics. Univariate analyses were performed to evaluate the association between change in visual functioning and disease category as well as wearing status and disease category. For all tests, a p value $<0.05$ was used to determine statistical significance. Data analyses were conducted using SAS (V.9.1) software for Windows (SAS Institute, Cary, North Carolina, USA).

\section{RESULTS}

As presented in figure 1, of the 199 patients in this cohort, 16 patients died by July 2013, and were excluded from subsequent analysis. Twenty-one patients were classified as non-candidates at consultation, although no patients were excluded because of disease severity. Twenty-six patients did not complete the customisation and training process. There were 15 patients lost to follow-up for whom wearing status could not be ascertained. There was no significant difference in primary indication for treatment between candidates and non-candidates $\left(p=0.303, \chi^{2}\right)$.

Of the remaining 121 patients, there were 65 females $(53.7 \%)$ and 56 males (46.3\%). The mean age was 51.57 years $(\mathrm{SD}=15$, range $8-78)$. The OSD group included patients with dry eye syndrome, limbal stem cell deficiency, epidermal ocular disorders, neurotrophic keratitis and corneal exposure. The specific diagnoses represented most frequently were dry eye syndrome, ocular chronic GVHD and Stevens-Johnson syndrome. The distorted corneal surface group included patients with corneal degenerations, corneal dystrophy, history of corneal surgery and corneal scar. The specific diagnoses represented most frequently were keratoconus, irregular cornea after Penetrating Keratoplasty (PK) and irregular cornea after LASIK.

There was a preponderance of males (M:F of 32:25) among patients with distorted corneal surface (mean age 50.7) and females (M:F of 24:40) among patients with OSD (mean age 52.4). At 5-year follow-up, continued device wear was confirmed in $89 / 121(73.6 \%)$ patients. Discontinuation of wear was confirmed in 32/121 (26.4\%) patients; the most common reasons for discontinuation are reported in table 1.

Ophthalmic complications of PROSE treatment were uncommon and were recorded as the reason for discontinuation of wear in 3/121 (2.5\%) patients. The complications were recorded as 'redness', 'corneal graft failure and elevated pressure', and 'opacification'.

A significant relationship exists between wearing status at 5 years and primary disease category $(p=0.0079$, two-tailed $t$ test). There was an increased likelihood of continued device wear at 5 years in patients with distorted corneal surface (84\%) compared with those with OSD (64\%), $(p=0.0121$, $\left.\chi^{2}\right)$. Further analysis reveals that there was only one subgroup of patients with OSD, those with ocular chronic graftversus-host disease $(n=12)$, that had a 5 -year wearing rate (75\%) equal to that of patients with distorted corneal surface $\left(p=0.4442, \chi^{2}\right)$. Potential confounding associations were analysed and it was found that there was no statistically significant relationship between continued device wear at 5 years and age, gender, proximity to the Boston Foundation for Sight, or change in general health status as measured from the NEI VFQ from baseline to 5 years $(p>0.05$, for each mean, by unpaired t test).

Figure 2 demonstrates that NEI VFQ-25 composite scores increased for patients wearing PROSE devices at 6 months $(\Delta=$ +23 points, mean $=82, \mathrm{p}<0.001$, two-tailed t test) with no significant decline among those still wearing a device at 5 years ( $\Delta=-4$ points, mean $=78, p=0.22$, two-tailed t test). We found no significant relationship between baseline VFQ-25 score and wearing status at 5 years $(p=0.8602$, two-tailed test $)$ and no significant relationship between baseline General Health score and wearing status at 5 years $(p=0.5280$, two-tailed $t$ test $)$, thus indicating that initial VFQ-25 and general health scores of patients who are dispensed devices are not predictive of future long-term wear. The mean change in general health VFQ score from baseline to 5 years was not significantly different among

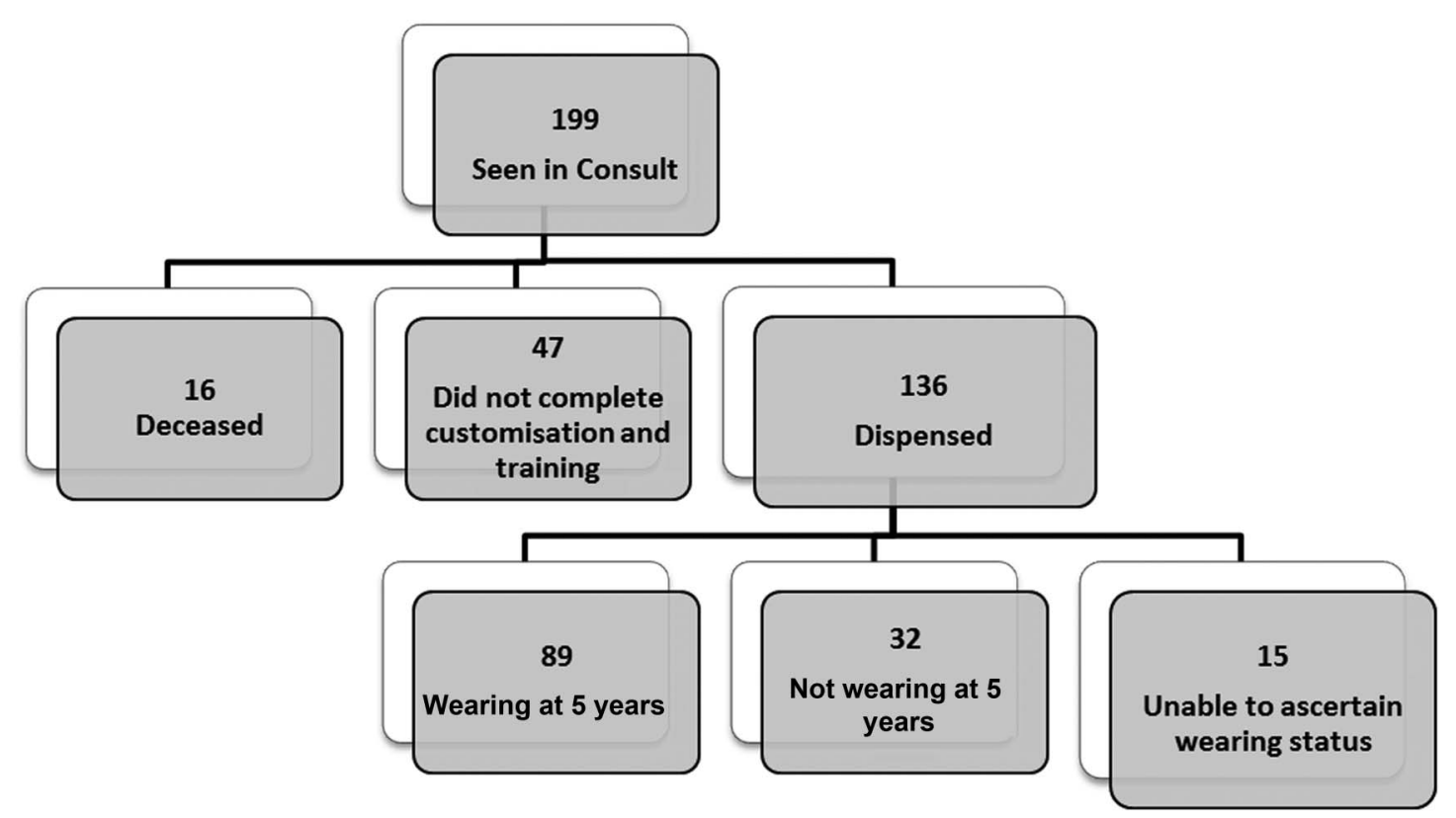

Figure 1 Candidacy for prosthetic replacement of the ocular surface ecosystem (PROSE) treatment, device dispensed status and 5-year wearing status. 
Table 1 Reasons for discontinuation of wear

\begin{tabular}{llll}
\hline Reason & $\begin{array}{l}\text { Number of } \\
\text { patients }\end{array}$ & $\begin{array}{l}\text { Distorted } \\
\text { surface:OSD }\end{array}$ & $\begin{array}{l}\text { Mean duration } \\
\text { of wear (years) }\end{array}$ \\
\hline Discomfort & 7 & $4: 3$ & 0.6 \\
Limited improvement of VA & 5 & $1: 4$ & 2.2 \\
Insertion/removal too difficult & 4 & $1: 3$ & 0.8 \\
Cured & 4 & $2: 2$ & 2.3 \\
Ophthalmic complications & 3 & $2: 1$ & 0.7 \\
'Stopped working' & 3 & $0: 3$ & 0.7 \\
Lost/broken & 2 & $1: 1$ & 4.9 \\
Disease progression & 2 & $0: 2$ & 0.8 \\
Cosmetic issues & 1 & $0: 1$ & 0.2 \\
Systemic disease & 1 & $0: 1$ & 3.9 \\
\hline
\end{tabular}

Median duration of wear before discontinuation was 1.0 year. There is no relationship between reasons stopped wearing and primary diagnosis $(r=-0.02145, p=0.9072)$. OSD, ocular surface disease; VA, visual acuity. patients who continued to wear devices $(p=0.9090)$ and those who did not $(\mathrm{p}=0.1183)$. The VFQ-25 scores broken down by disease category and wearing status are found in table 2 .

There was no significant relationship between the change in VFQ-25 score from baseline to 5-year follow-up and disease category (using analysis of variance (ANOVA) procedure: $\mathrm{R}^{2}=0.002034, \mathrm{p}=0.6462$ ). Furthermore, there was no significant relationship between the change in VFQ-25 score from baseline to 5-year follow-up and disease category within just those patients who continued to wear at 5 years (using ANOVA procedure: $\mathrm{R}^{2}=0.00520, \mathrm{p}=0.8440$ ). Likewise, there was no significant relationship between the change in VFQ-25 score from baseline to 5-year follow-up and disease category within just those patients who discontinued wear (using ANOVA procedure: $\mathrm{R}^{2}=0.001358, \mathrm{p}=0.8495$ ).

Within the 32 patients who discontinued wear of PROSE device, the median time until discontinuation was 1 year. A smaller increase in visual function with PROSE treatment at
Figure 2 Graph showing Visual Function Questionnaire (VFQ-25) changes in regard to wearing status. Those wearing had significant improvement in visual function at 6 months $(\Delta=+23$ points, $p<0.001$, mean $=82, n=87$ ) with no significant decline at 5 years $(\Delta=-4$ points, $p=0.22$, mean $=78, n=89$ ). Those no longer wearing at 5 years had improvement in visual function at 6 months $(\Delta=+14$ points, $\mathrm{p}=0.08$, mean $=72, n=32$ ) with no significant decline at 5 years as well $(\Delta=-2$ points, $p=0.65$, mean $=70, n=32$ ). However, the magnitude of the change for the no longer wearing group was not as great. Further, there remained significant difference $(p=0.029)$ in visual function (8 points) between those wearing at 5 years and those no longer wearing.

\section{Wearing at 6 months and Five years}

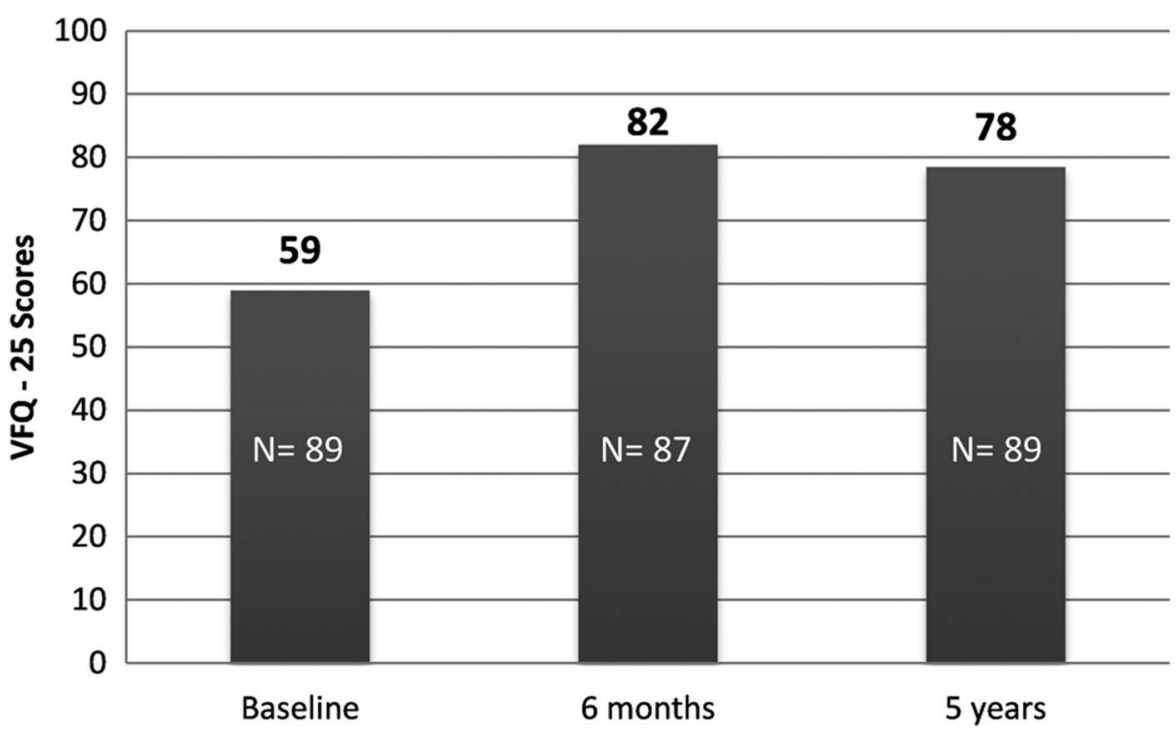

No Longer Wearing at Five years

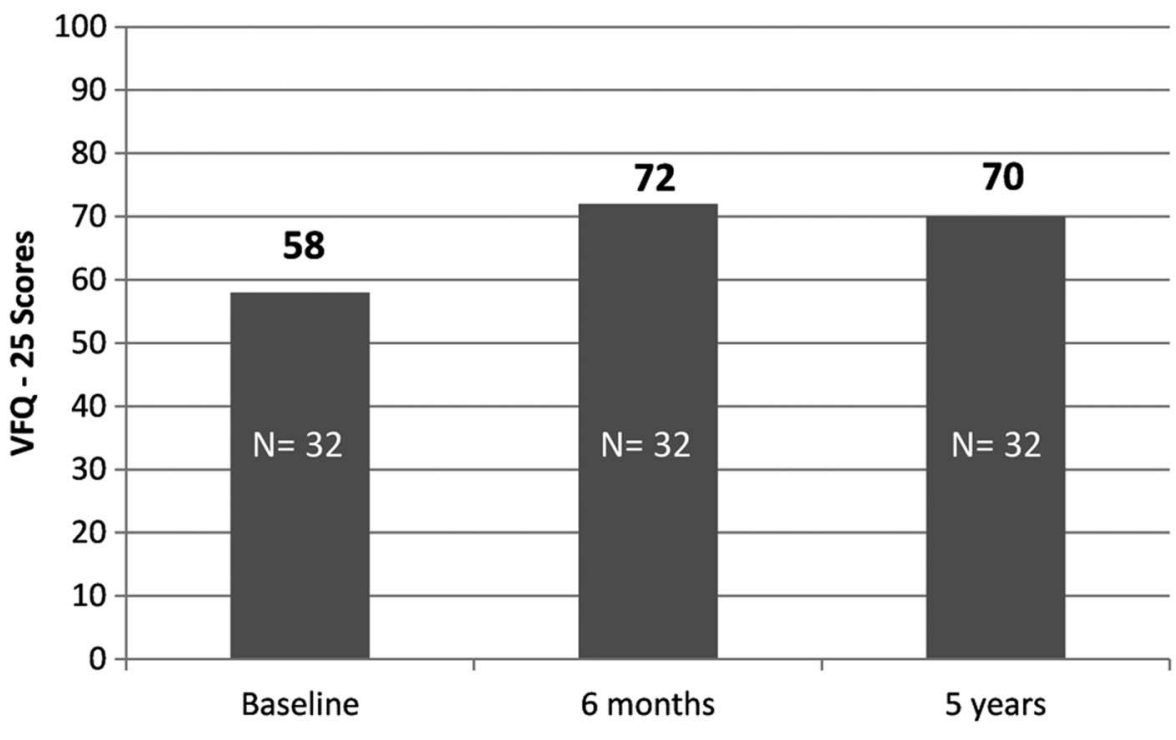


Table 2 VFQ-25 scores delineated by wearing status and primary disease category: distorted corneal surface and ocular surface disease (OSD)

\begin{tabular}{|c|c|c|c|c|c|c|}
\hline \multirow[b]{2}{*}{ Wearing at 5 years? } & \multicolumn{2}{|c|}{ Distorted corneal surface } & \multicolumn{2}{|l|}{ OSD } & \multicolumn{2}{|l|}{ Total } \\
\hline & $\begin{array}{l}\text { Yes } \\
\mathrm{N}=48\end{array}$ & $\begin{array}{l}\text { No } \\
\mathrm{N}=9\end{array}$ & $\begin{array}{l}\text { Yes } \\
\mathrm{N}=41\end{array}$ & $\begin{array}{l}\text { No } \\
\mathrm{N}=23\end{array}$ & $\begin{array}{l}\text { Yes } \\
\mathrm{N}=89\end{array}$ & $\begin{array}{l}\text { No } \\
\mathrm{N}=32\end{array}$ \\
\hline Baseline VFQ-25 score & 62.40 & 64.44 & 54.90 & 55.83 & 58.94 & 58.25 \\
\hline 6-month VFQ-25 score & 86.00 & 68.67 & 82.07 & 73.67 & 84.42 & 72.24 \\
\hline 5-year VFQ-25 score & 82.64 & 76.91 & 73.70 & 67.03 & 78.46 & 69.75 \\
\hline 5-year change VFQ-25 score & +20.24 & +12.47 & +18.80 & +11.20 & +19.52 & +11.5 \\
\hline Baseline GHS score & 68.75 & 61.11 & 57.32 & 59.78 & 63.48 & 60.15 \\
\hline 6-month GHS score & 73.08 & 66.67 & 68.52 & 53.33 & 71.24 & 57.14 \\
\hline 5-year GHS score & 68.90 & 50.00 & 60.42 & 52.38 & 64.94 & 51.72 \\
\hline 5-year change GHS score & +0.15 & -11.11 & +3.10 & -7.40 & +1.46 & -8.43 \\
\hline
\end{tabular}

VFQ-25, NEI VFQ-25 composite score, Visual Function Questionnaire's main categories; GHS, NEI VFQ self-reported general health status.

6 months is associated with discontinuation of wear, and this group shows no further decline in visual function at 5 years.

\section{DISCUSSION}

The purpose of this study was to determine the impact of PROSE treatment at 5 years. The main outcome measures were wearing status and change in visual function at 5 years. This study found that $89 / 121(73.6 \%)$ of patients who complete PROSE treatment were still wearing devices at 5 years and that these patients experienced significant improvement in visual functioning at 6 months that was sustained at 5 years with continued device wear.

It is notable that the patients referred to this centre and described in this report have high disease severity with significant impact on visual function; mean baseline VFQ-25 score is just under 60 points. Furthermore, no candidates were excluded from treatment based on disease severity. The 5-year wearing rate of $89 / 121(73.6 \%)$ patients was calculated without including the 4/121 (3.3\%) patients who reported a 'cure' and without including the two patients who lost or broke their devices at time of follow-up but who were planning on resuming wear. If we considered these data then the success rate of PROSE treatment at 5 years, as defined by continued wearing status or cessation of disease state, is 95/121 (78.5\%). Of note, the 15 patients who were lost to follow-up had indeterminate wearing status, leaving the possibility that their true wearing status, if it could be ascertained, would increase or decrease the 5 -year success rate of PROSE treatment, as well as the rate of complications.

Improvement in visual function at 6 months is associated with continuation of wear by 5 years; the magnitude of the initial impact of PROSE treatment is, not surprisingly, a predictor of continued wear. Likewise, the failure of those who discontinued wear to experience significant decline in visual function suggests that PROSE treatment was undertaken during a low period in visual function, and that with regression to the mean, visual function improved independent of this intervention. There was a clinically significant difference in wearing status at 5 years between the distorted cornea and OSD group. In a 2010 report on a 2006 cohort from this centre, ${ }^{4}$ Stason et al found that there was greater improvement in logMAR visual acuity among the distorted cornea group than the OSD group; it may be that magnitude of change in visual acuity correlates with continuation of wear. Alternatively, it is possible that a period of PROSE device wear allowed for sufficient healing of the ocular surface to maintain ocular surface integrity even after discontinuation of lens wear. Finally, our clinical experience is that patients with a neuralgic basis for dry eye syndrome are less likely to continue with wear. ${ }^{13}$

The GVHD subgroup still living at 5 years had a wearing rate of $75 \%$ which is statistically equivalent to that of patients with distorted cornea. Patients with GVHD were more likely to continue wear than patients with other OSDs. Six of the sixteen deceased patients excluded from the analysis due to lack of 5 -year follow-up data had ocular chronic GVHD and likely would increase the 5-year success rate had they lived. Statistical analysis and discussion of other specific OSD and distorted cornea subgroups is limited by the small sample size in each subgroup.

After 5 years, the magnitude of change in visual function from baseline observed across all diagnoses in this cohort is +19 points which is consistent with previous reports on PROSE treatment. $^{4} 9$ It is meaningful to consider that changes in VFQ-25 score of 5-10 points in a study of this sample size are considered clinically significant enough to detect differences between two self-selected groups with a repeated measure design (assume $\alpha=0.05$, two-tailed t test, power $=80 \%$ and an intertemporal correlation between scores of 0.60). ${ }^{14}$ Moreover, in many corneal diseases like keratoconus, visual function tends to decline over time, ${ }^{15}$ further highlighting the long-term therapeutic impact of PROSE treatment reported here.

The results in table 2 indicate that there is some interplay between self-reported general health status and continued wear of PROSE device; in those still wearing PROSE devices after 5 years, there was a continued improvement in general health, whereas in those who ceased wearing there was a reported decline. A causal relationship between general health status and continued device wear could be explored in future studies.

Keratoplasty is typically considered the next step after contact lens failure for patients with irregular corneal surface. In a 1994 report from Bourne et al, ${ }^{16}$ the overall success of keratoplasty at 5 years can be calculated to be $57 \%$ based on the sum of percentages failing by rejection, failure and regraft. Data from the Australian corneal graft registry analysed 18686 penetrating corneal grafts and found that the probability of corneal graft survival was 0.73 at 5 years. ${ }^{17}$ This success rate is reported in terms of clear graft and not in terms of visual function. It is worth noting here that an important study by Mendes et al ${ }^{18}$ showed that impact of grafting on VFQ is inversely proportional to vision in the fellow eye, and thus that impact on function may be minimal if the fellow eye has good vision. It is important to study patients, not eyes, which we have done. Towards this end, DeLoss et al used the OSD Index (OSDI), which is a global rather than eye specific metric, in their recent study of outcome of PROSE treatment versus keratoplasty at a single institution. 
These investigators found better 1-year outcome on acuity and OSDI and fewer complications in PROSE treatment when compared with keratoplasty. ${ }^{19}$ PROSE treatment compares favourably with keratoplasty in economic analysis as well. PROSE treatment is substantially more cost effective at \$24 900/Quality Adjusted Life Year (QALY) ${ }^{11}$ when compared with PK at \$56 409/QALY and Descemet's Stripping Endothelial Keratoplasty (DSEK) at 42 904/QALY in a 2013 analysis. ${ }^{20}$ Many analyses fail to incorporate the long-term burden of maintaining the effect of surgical or medical intervention, as well as possibility of complication which are high for keratoplasty. An alternative option such as PROSE treatment warrants consideration and further comparative study. Current advances in keratoplasty for keratoconus, such as deep anterior lamellar keratoplasty, may improve functional outcomes, but until that data emerges it seems that the long-term impact of PROSE treatment compares favourably with keratoplasty for the distorted corneal surface.

Limitations of this study are that it is retrospective and observational. A randomised controlled trial or a case-control study would be required to determine if PROSE treatment is specifically the reason for sustained improvement in visual function. Because the NEI VFQ-25 evaluates visual function with respect to the patient and not the individual eye, there may be factors other than PROSE treatment that have resulted in a positive or negative change in visual function. As mentioned above, the sample size of this study did not allow meaningful statistical analysis among many specific diagnostic subgroups within the OSD or distorted cornea groups.

In summary, PROSE treatment offers continued long-term benefit, as defined by continued device wear and improved visual functioning at 5 years, in patients with complex corneal disease.

Contributors JSA: data acquisition/analysis/interpretation, drafting and revising of work, final approval. NRK: data analysis/interpretation of data, drafting and revising of work, final approval. DSJ: study conception, design, interpretation of data, drafting and revising, final approval.

Competing interests DSJ is a salaried, full-time employee of the non-profit Boston Foundation for Sight, 501(c)3.

Ethics approval New England Institutional Review Board.

Provenance and peer review Not commissioned; externally peer reviewed.

Open Access This is an Open Access article distributed in accordance with the Creative Commons Attribution Non Commercial (CC BY-NC 4.0) license, which permits others to distribute, remix, adapt, build upon this work non-commercially, and license their derivative works on different terms, provided the original work is properly cited and the use is non-commercial. See: http://creativecommons.org/ licenses/by-nc/4.0/

\section{REFERENCES}

1 Cotter JM, Rosenthal P. Scleral contact lenses. J Am Optom Assoc 1998;69:33-40.

2 Romero-Rangel T, Stavrou P, Cotter J, et al. Gas-permeable scleral contact lens therapy in ocular surface disease. Am J Ophthalmol 2000;130:25-32.

3 Jacobs DS, Rosenthal P. Boston scleral lens prosthetic device for treatment of severe dry eye in chronic graft-versus-host disease. Cornea 2007;26:1195-9.

4 Stason WB, Razavi M, Jacobs DS, et al. Clinical benefits of the Boston Ocular Surface Prosthesis. Am J Ophthalmol 2010;149:54-61.

5 Schein OD, Rosenthal P, Ducharme C. A gas-permeable scleral contact lens for visual rehabilitation. Am J Ophthalmol 1990;109:318-22.

6 Takahide K, Parker PM, Wu M, et al. Use of fluid-ventilated, gas-permeable scleral lens for management of severe keratoconjunctivitis sicca secondary to chronic graft-versus-host disease. Biol Blood Marrow Transplant 2007;13:1016-21.

7 Papakostas TD, Le HG, Chodosh J, et al. Prosthetic Replacement of the ocular surface ecosystem as treatment for ocular surface disease in patients with a history of Stevens-Johnson syndrome/toxic epidermal necrolysis. Ophthalmology 2015; 122:248-53.

8 Heur M, Bach D, Theophanous C, et al. Prosthetic replacement of the ocular surface ecosystem scleral lens therapy for patients with ocular symptoms of chronic Stevens-Johnson Syndrome. Am J Ophthalmol 2014;158:49-54.

9 Baran I, Bradley JA, Alipour F, et al. PROSE treatment of corneal ectasia. Cont Lens Anterior Eye 2012;35:222-7.

10 Gungor I, Schor K, Rosenthal P, et al. The Boston Scleral Lens in the treatment of pediatric patients. J AAPOS 2008;12:263-7.

11 Shepard DS, Razavi M, Stason WB, et al. Economic appraisal of the Boston Ocular Surface Prosthesis. Am J Ophthalmol 2009;148:860-8.e2.

12 Mangione CM, Lee PP, Gutierrez PR, et al. Development of the 25-item National Eye Institute Visual Function Questionnaire. Arch Ophthalmol 2001;119:1050-8.

13 Theophanous C, Jacobs DS, Hamrah P. Corneal Neuralgia after LASIK. Optom Vis Sci 2015;92:e233-40.

14 The National Eye Institute 25-Item Visual Function Questionnaire (VFQ-25). 2000. http://www.nei.nih.gov/resources/visionfunction/manual_cm2000.pdf (accessed 31 Dec 2013).

15 Kymes SM, Walline JJ, Zadnik K, et al. Changes in the quality-of-life of people with keratoconus. Am J Ophthalmol 2008;145:611-17.

16 Bourne WM, Hodge DO, Nelson LR. Corneal endothelium five years after transplantation. Am J Ophthalmol 1994;118:185-96.

17 Williams KA, Lowe $M$, Bartlett $C$, et al. Risk factors for human corneal graft failure within the Australian corneal graft registry. Transplantation 2008;86:1720-4.

18 Mendes F, Schaumberg DA, Navon S, et al. Assessment of visual function after corneal transplantation: the quality of life and psychometric assessment after corneal transplantation (Q-PACT) study. Am J Ophthalmol 2003;135:785-93.

19 DeLoss KS, Fatteh NH, Hood CT. Prosthetic Replacement of the Ocular Surface Ecosystem (PROSE) scleral device compared to keratoplasty for the treatment of corneal ectasia. Am J Ophthalmol 2014;158:974-82.

20 Bose S, Ang M, Mehta JS, et al. Cost-effectiveness of Descemet's stripping endothelial keratoplasty versus penetrating keratoplasty. Ophthalmology 2013;120:464-70. 